\title{
Lessons of spin and torsion: Reply to "Consistent coupling to Dirac fields in teleparallelism"
}

\author{
Yu. N. Obukhov* \\ Institute for Theoretical Physics, University of Cologne, 50923 Köln, Germany
}

J. G. Pereira

Instituto de Física Teórica, Universidade Estadual Paulista, Rua Pamplona 145, 01405-900 São Paulo SP, Brazil

(Received 15 October 2003; published 2 June 2004)

\begin{abstract}
In reply to the criticism made by Mielke in the preceding Comment on our recent paper, we once again explicitly demonstrate the inconsistency of the coupling of a Dirac field to gravitation in the teleparallel equivalent of general relativity. Moreover, we stress that the mentioned inconsistency is generic for all sources with spin and is by no means restricted to the Dirac field. In this sense the $S L(4, R)$-covariant generalization of the spinor fields in the teleparallel gravity theory is irrelevant to the inconsistency problem.
\end{abstract}

DOI: 10.1103/PhysRevD.69.128502

PACS number(s): 04.50. $+\mathrm{h}, 03.50 . \mathrm{Kk}, 04.20 . \mathrm{Jb}$

\section{INTRODUCTION}

The problem of coupling sources of spinning matter to the teleparallel gravitational field is well known; see [1-4], for example. This difficulty is naturally related to the fact that teleparallelism can be consistently treated as the gauge theory of the translation group of spacetime. The corresponding dynamical current, for the generators of translations, is the energy-momentum. Accordingly, the teleparallelism is perfectly equivalent to the general relativity theory for matter sources without spin. However, as is well known, the spin current corresponds to the generators of the Lorentz group and in this sense it does not formally "fit" into the gauge approach based on the group of translations. This is different from the more general gauge theory based on the Poincare symmetry group (semidirect product of translations times the Lorentz group) in which the energy-momentum and the spin currents have equal "rights," and in which they are consistently coupled to the curvature and torsion of spacetime.

In our recent paper [5], we developed a metric-affine approach to teleparallel gravity in which the latter was treated as a particular case of general metric-affine gravity (MAG) specified by the geometric constraints of a vanishing curvature and nonmetricity. Among other results, this approach provided an explicit demonstration of the inconsistency of the coupling of matter fields with spin. The author of the Comment [6] tries to dispute this result. In our reply we show that their claim is misleading.

\section{INCONSISTENCY OF THE SPIN COUPLING}

Since this point seems to be a source of constant misunderstandings in studies of teleparallel gravity, we will clarify the corresponding result by using three different techniques.

\section{A. Tetrad approach}

First, let us recall that one can deal with teleparallelism in the purely tetrad framework by taking the coframe compo-

\footnotetext{
*On leave from Department of Theoretical Physics, Moscow State University, 117234 Moscow, Russia.
}

nents $h_{i}^{\alpha}$ as the basic field variables [7] and treating the torsion tensor $T^{k}{ }_{i j}=h_{\alpha}^{k}\left(\partial_{i} h_{j}^{\alpha}-\partial_{j} h_{i}^{\alpha}\right)$ as the translational gauge field strength $[2,3]$. The action, with a Yang-Mills type of Lagrangian, is

$$
S=\int d^{4} x h\left(\frac{1}{4 \kappa} S_{k}^{i j} T_{i j}^{k}+L^{\mathrm{mat}}\right),
$$

where $h=\operatorname{det} h_{i}^{\alpha}$ and $S^{k i j}=\frac{1}{4} T^{k i j}+\frac{1}{4}\left(T^{i k j}-T^{j k i}\right)-\frac{1}{2}\left(g^{k j} T^{l i}{ }_{l}\right.$ $\left.-g^{k i} T^{l j}{ }_{l}\right)$. The variation of the action with respect to the tetrad yields the field equation

$$
\frac{1}{h} \partial_{j}\left(h S_{k}^{i j}\right)+S_{l}^{m i} T_{m k}^{l}-\frac{1}{4} \delta_{k}^{i} S_{l}^{m n} T_{m n}^{l}=\kappa T_{k}^{i}
$$

The right-hand side is the canonical energy-momentum of matter defined by the variational derivative $T_{k}{ }^{i}$ $=h_{k}^{\alpha} \delta\left(h L^{\text {mat }}\right) /\left(h \delta h^{\alpha}{ }_{i}\right)$.

The inconsistency of the coupling of matter with spin arises as follows. The tetrad $h_{i}^{\alpha}$ has 16 independent components and, accordingly, Eq. (2) also has 16 components. However, there is a well-known geometric identity which relates the left-hand side to the Einstein tensor:

$$
\frac{1}{h} \partial_{j}\left(h S_{k}^{i j}\right)+S_{l}^{m i} T_{m k}^{l}-\frac{1}{4} \delta_{k}^{i} S_{l}^{m n} T_{m n}^{l} \equiv \widetilde{G}_{k}^{i}
$$

The tilde denotes the purely Riemannian object constructed from the spacetime metric $g_{i j}$. Since the Einstein tensor is symmetric, $\widetilde{G}_{i j}=\widetilde{G}_{j i}$, we immediately discover that the field equation (2) yields the vanishing of the antisymmetric part of the canonical energy-momentum tensor: $T_{[i j]}=0$. Using the Noether conservation law of total angular momentum, we then find that the spin tensor $\tau^{k}{ }_{i j}=-\tau^{k}{ }_{j i}$ must itself be conserved, $\nabla_{k} \tau^{k}{ }_{i j}=0$.

\section{B. MAG approach: First field equation}

The same result can be rederived within the framework of the MAG approach. Since that was the subject of our previous paper [5], we merely state here that the first field equa- 
tion (derived from the variation with respect to the coframe) reads, for the teleparallel equivalent Lagrangian,

$$
\frac{1}{2} \eta_{\alpha \mu \nu} \wedge \widetilde{R}^{\mu \nu}=\kappa \Sigma_{\alpha} .
$$

Here $\widetilde{R}^{\mu \nu}$ is the two-form of the Riemannian curvature, and $\Sigma_{\alpha}$ is the three-form of the canonical energy-momentum of matter. It is easy to verify that

$$
\vartheta_{[\alpha} \wedge \eta_{\beta] \mu \nu} \equiv-\eta_{\alpha \beta[\mu} \wedge \vartheta_{\nu]} .
$$

As a result, we straightforwardly see that the antisymmetric part of the left-hand side of Eq. (4) vanishes, $\vartheta_{[\alpha} \wedge \eta_{\beta] \mu \nu}$ $\wedge \widetilde{R}^{\mu \nu}=-\eta_{\alpha \beta \mu} \wedge \vartheta_{\nu} \wedge \widetilde{R}^{\mu \nu} \equiv 0$, in view of the Ricci identity $-\widetilde{R}_{\mu}{ }^{\nu} \wedge \vartheta_{\nu}=\widetilde{D} \widetilde{D} \vartheta_{\mu}=0$.

Consequently, we again find that the antisymmetric part of the energy-momentum current must vanish, $\vartheta_{[\alpha} \wedge \Sigma_{\beta]}=0$, and hence the spin two-form $\tau_{\alpha \beta}=\tau_{\alpha \beta}^{k} \eta_{k}$ should be conserved: $D \tau_{\alpha \beta}=0$.

\section{MAG approach: Second field equation}

Finally, let us prove the above result by following the same reasoning as the author of the Comment [6] who analyzed the second field equation. It reads [see Eq. (4.3) of [6]]

$$
D \lambda_{\alpha \beta}+\vartheta_{[\alpha} \wedge H_{\beta]}^{\|}=\tau_{\alpha \beta} .
$$

Since the teleparallelism equivalent translational momentum is given by $H_{\alpha}^{\|}=\left(1 / \ell^{2}\right) \eta_{\alpha \mu \nu} \wedge K^{\mu \nu}$ in terms of the contortion one-form $K^{\mu \nu}$, we have

$$
\vartheta_{[\alpha} \wedge H_{\beta]}^{\|} \equiv-\frac{1}{\ell^{2}} D \eta_{\alpha \beta} .
$$

Indeed, using the identity (5), we find $\vartheta_{[\alpha} \wedge \eta_{\beta] \mu \nu} \wedge K^{\mu \nu}$ $=-\eta_{\alpha \beta \mu} \wedge \vartheta_{\nu} \wedge K^{\mu \nu}=-\eta_{\alpha \beta \mu} \wedge T^{\mu}$. Then the above result is easily found with the help of the general formulas (3.8.5) of [8] which give the covariant derivatives of the $\eta$-forms. Substituting Eq. (7) into Eq. (6) and subsequently taking the covariant exterior derivative, we obtain $D \tau_{\alpha \beta}=0$, since $D D\left(\lambda_{\alpha \beta}-\eta_{\alpha \beta} / \ell^{2}\right)=0$ in view of the teleparallel constraint requiring the vanishing of the total curvature.

Thus, Eq. (4.6) of the Comment [6] is totally misleading in the sense that a clear zero is "hidden" in the second term on the left-hand side.

\section{MAKING SPIN COUPLING CONSISTENT}

We have demonstrated above that the gravitational coupling of spin is generically inconsistent in teleparallel equivalent gravity. How can one cure this situation? The source of the difficulty is clear: the left-hand side (geometric one) of the gravitational field equation is symmetric, whereas the right-hand side (source) is asymmetric for matter with spin. Correspondingly, one can proceed in one of two ways: (i) introduce a different coupling rule so that the energymomentum becomes symmetric, or (ii) change the dynamical scheme so that the geometric left-hand side also becomes asymmetric.

\section{A. Alternative coupling prescription: Einstein's theory}

In [4], and more recently in [9] (see also the earlier discussion in [1]), it was noticed that if the coupling Lagrangian of a spinor field contains not the Weitzenböck connection of teleparallelism but the usual Riemannian connection, then the coupling inconsistency disappears. In this case, Eq. (4) is replaced by

$$
\frac{1}{2} \eta_{\alpha \mu \nu} \wedge \widetilde{R}^{\mu \nu}=\kappa \sigma_{\alpha} .
$$

The three-form $\sigma_{\alpha}$ on the right-hand side is the so-called Belinfante-Rosenfeld energy-momentum. It is symmetric, $\vartheta_{[\alpha} \wedge \sigma_{\beta]}=0$. Consequently, there is no coupling inconsistency. The teleparallel gravity with such a coupling prescription becomes indistinguishable from Einstein's general relativity theory.

\section{B. From translations to Poincaré group: Einstein-Cartan theory}

An alternative procedure is to include the spin, together with the energy-momentum current, as a dynamical source of equal right for the gravitational field. This naturally leads to the gauge theory based on the Poincare symmetry group with the generators of translations related to the canonical energymomentum $\Sigma_{\alpha}$, and the generators of the Lorentz group related to spin $\tau_{\alpha \beta}$. Such an extension of the dynamical content yields an extension of the spacetime geometry to the Riemann-Cartan case with nontrivial curvature $R_{\alpha}{ }^{\beta}$ and torsion $T^{\alpha}$ two-forms. The extended Einstein-Hilbert Lagrangian then yields the Einstein-Cartan field equations

$$
\begin{gathered}
\frac{1}{2} \eta_{\alpha \mu \nu} \wedge R^{\mu \nu}=\kappa \Sigma_{\alpha}, \\
\frac{1}{2} \eta_{\alpha \beta \mu} \wedge T^{\mu}=\kappa \tau_{\alpha \beta} .
\end{gathered}
$$

This system is completely consistent in the sense that the antisymmetric part of Eq. (9), combined with the second equation (10), yields the Noether identity $D \tau_{\alpha \beta}+\vartheta_{[\alpha} \wedge \Sigma_{\beta]}$ $=0$.

\section{DISCUSSION}

In the recent Comment on our paper [6], it was claimed that the spinor field couples consistently to the teleparallel gravitational field. However, this is incorrect and we have presented at least three direct demonstrations of the inconsistency of the spin coupling in the teleparallel equivalent gravity model.

(1) In simple terms, the mentioned inconsistency arises from the fact that the left-hand side of the teleparallel gravitational field equation is symmetric,whereas the right-hand side is represented by the canonical energy-momentum, 
which is nonsymmetric for matter with spin.

(2) The inconsistency is generic, i.e., it is not specific for the Dirac spinor field, but rather concerns all sources with spin. In this sense, the remark of [6] about the necessity of considering more general $S L(4, R)$-covariant multispinors is irrelevant. The spin coupling inconsistency will be present for such matter as well.

(3) The well-known fact that the teleparallel equivalent Lagrangian differs from the Einstein-Hilbert Lagrangian by a total derivative $[8,10]$ is also irrelevant for the demonstration of the coupling inconsistency. Neither in our original paper [5] nor in the derivations above did we ever need or use that fact.
We have shown that, contrary to the erroneous statement of [6], the coupling of spin can be made consistent only either by a change of the coupling prescription (thereby formally obtaining a description equivalent to general relativity) or by a change of the dynamical scheme (thus arriving at the Einstein-Cartan gravity theory).

\section{ACKNOWLEDGMENTS}

The work of Y.N.O. was supported by the Deutsche Forschungsgemeinschaft (Bonn) with the grant HE 528/20-1. J.G.P. thanks FAPESP and CNPq for partial financial support. We are grateful to Friedrich Hehl for helpful comments.
[1] K. Hayashi and T. Shirafuji, Phys. Rev. D 19, 3524 (1979).

[2] V.C. de Andrade and J.G. Pereira, Phys. Rev. D 56, 4689 (1997).

[3] V.C. de Andrade, L.C.T. Guillen, and J.G. Pereira, Phys. Rev. Lett. 84, 4533 (2000).

[4] V.C. de Andrade, L.C.T. Guillen, and J.G. Pereira, Phys. Rev. D 64, 027502 (2001).

[5] Yu.N. Obukhov and J.G. Pereira, Phys. Rev. D 67, 044016 (2003)
[6] E.W. Mielke, preceding Comment, Phys. Rev. D 69, 128501 (2004).

[7] Our notation and conventions are as in $[5,8]$. In particular, the Greek indices $\alpha, \beta, \ldots$ label the anholonomic frame components (e.g., $\vartheta^{\alpha}$ ), and the Latin indices $i, j, \ldots$ label the local spacetime coordinates (e.g., $x^{i}$ and $d x^{i}$ ).

[8] F.W. Hehl, J.D. McCrea, E.W. Mielke, and Y. Ne'eman, Phys. Rep. 258, 1 (1995).

[9] J.W. Maluf, Phys. Rev. D 67, 108501 (2003).

[10] Yu.N. Obukhov, E.J. Vlachynsky, W. Esser, and F.W. Hehl, Phys. Rev. D 56, 7769 (1997). 